\title{
Is the Composite Fermion a Dirac Particle?
}

\author{
Dam Thanh Son \\ Kadanoff Center for Theoretical Physics, University of Chicago, Chicago, Illinois 60637, USA
}

(Received 19 February 2015; published 2 September 2015)

\begin{abstract}
We propose a particle-hole symmetric theory of the Fermi-liquid ground state of a half-filled Landau level. This theory should be applicable for a Dirac fermion in the magnetic field at charge neutrality, as well as for the $\nu=\frac{1}{2}$ quantum Hall ground state of nonrelativistic fermions in the limit of negligible interLandau-level mixing. We argue that when particle-hole symmetry is exact, the composite fermion is a massless Dirac fermion, characterized by a Berry phase of $\pi$ around the Fermi circle. We write down a tentative effective field theory of such a fermion and discuss the discrete symmetries, in particular, $\mathcal{C P}$. The Dirac composite fermions interact through a gauge, but non-Chern-Simons, interaction. The particle-hole conjugate pair of Jain-sequence states at filling factors $n /(2 n+1)$ and $(n+1) /(2 n+1)$, which in the conventional composite fermion picture corresponds to integer quantum Hall states with different filling factors, $n$ and $n+1$, is now mapped to the same half-integer filling factor $n+\frac{1}{2}$ of the Dirac composite fermion. The Pfaffian and anti-Pfaffian states are interpreted as $d$-wave Bardeen-Cooper-Schrieffer paired states of the Dirac fermion with orbital angular momentum of opposite signs, while $s$-wave pairing would give rise to a particle-hole symmetric non-Abelian gapped phase. When particle-hole symmetry is not exact, the Dirac fermion has a $\mathcal{C} \mathcal{P}$-breaking mass. The conventional fermionic Chern-Simons theory is shown to emerge in the nonrelativistic limit of the massive theory.
\end{abstract}

DOI: 10.1103/PhysRevX.5.031027

\section{INTRODUCTION}

The theory of the fractional quantum Hall (FQH) effect $[1,2]$ is based on the paradigm of the composite fermion [3-5], which provides a unified explanation of a large amount of observed phenomena, among which the most early ones are the Jain sequences-series of quantum Hall plateaux at filling factors $\nu$ near $1 / 2,1 / 4$, etc. The composite fermion picture gives rise to extremely accurate wave functions of $\mathrm{FQH}$ ground states [6].

At half filling, the composite fermion (CF) picture, developed into a mathematical framework of the ChernSimons (CS) field theory by Halperin, Lee, and Read (HLR) [5], predicts that the ground state is a Fermi liquid, providing an explanation for the results of acoustic-wavepropagation experiments [7]. Near, but not exactly at half filling, the CF is predicted to feel a small residual magnetic field, and the semiclassical motion of the $\mathrm{CF}$ in such a field has been observed experimentally [8,9]. The composite fermion theory also provides an elegant interpretation of the Pfaffian (or Moore-Read) state [10] as a $p_{x}+i p_{y}$ Bardeen-Cooper-Schrieffer (BCS) paired state [11].

Despite its success, one of the symmetries of $\mathrm{FQH}$ systems in the limit of zero Landau-level (LL) mixing-the

Published by the American Physical Society under the terms of the Creative Commons Attribution 3.0 License. Further distribution of this work must maintain attribution to the author(s) and the published article's title, journal citation, and DOI.
Subject Areas: Condensed Matter Physics

particle-hole symmetry [12]-is not explicit within the CF paradigm. The CF is constructed by attaching a magnetic flux to the electron before projecting to the lowest Landau level (LLL); the CS field theory formalism, strictly speaking, does not allow one to attach fluxes to holes in the LLL. In one manifestation of the particle-hole asymmetric nature of the formalism, the two Jain-sequence states with $\nu=n /(2 n+1)$ and $\nu=(n+1) /(2 n+1)$, which form a particle-hole conjugate pair, receive slightly different interpretations in the CF language: The former fraction is an integer quantum Hall (IQH) state of CFs with $n$ filled Landau levels, while in the latter, $n+1$ Landau levels are filled. A related issue of the CF picture is its failure to account for the anti-Pfaffian state $[13,14]$ - the particlehole conjugate of the Moore-Read state-in a simple manner.

The zero bare mass limit, where particle-hole symmetry is exact, is particularly difficult to analyze within the HLR theory. Kivelson et al. [15] analyzed the HLR theory at $\nu=\frac{1}{2}$ and found that particle-hole symmetry requires the liquid of CFs to have a Hall conductivity $\sigma_{x y}^{\mathrm{CF}}=-\frac{1}{2}$. In a zero net magnetic field, this means that the $\mathrm{CF}$ liquid has an anomalous Hall coefficient and seems to contradict the Fermi liquid nature of the CFs. Kivelson et al. did not find any set of Feynman diagrams that could lead to a nonzero $\sigma_{x y}^{\mathrm{CF}}$. As one possible solution, they proposed that the problem lies in the noncommutativity of the limit of $m \rightarrow 0$ (the LLL limit) and the limit of taking the density of impurities to zero (the clean limit). This proposal leaves 
unanswered the question of why $\sigma_{x y}^{\mathrm{CF}}$ is exactly $-\frac{1}{2}$ at all frequencies, independent of the physics of impurities. Few attempts have been made to resolve the apparent inability to fit particle-hole symmetry to the CF picture; examples include Ref. [16].

One logical possibility is that particle-hole symmetry is spontaneously broken and the HLR theory describes only one of the two $\nu=\frac{1}{2}$ ground states, which become degenerate at zero Landau level mixing. In this case, the anti-Pfaffian state would be inaccessible from within the HLR theory. Numerical simulations, however, seem to be consistent with a particle-hole symmetric $\nu=\frac{1}{2}$ ground state [17]. Away from half filling, despite the apparent asymmetry in the treatments of the $\nu=n /(2 n+1)$ and $\nu=(n+1) /(2 n+1)$ states, particle-hole symmetry maps one CF trial wave function to another with high accuracy [18]. In addition, a recent experiment, set up to measure the Fermi momentum using commensurability effects in a periodic potential, implies that the Fermi momentum is determined by the density of particles at $\nu<\frac{1}{2}$ and of holes at $\nu>\frac{1}{2}$ [19]. One interpretation of this experiment is that the $\nu=\frac{1}{2}$ state allows two alternative, but equivalent, descriptions as a Fermi liquid of either particles or holes. This would mean that the $\nu=\frac{1}{2}$ ground state coincides with its particle-hole conjugate.

The problem of particle-hole symmetry is more acute for systems with Dirac fermions. The Dirac fermion is realized in graphene and on the surface of topological insulators (TIs) and is a relatively new venue for studying the quantum Hall $(\mathrm{QH})$ effect. For Dirac fermions, the IQH plateaux occur at half-integer values of the Hall conductivity: $\sigma_{x y}=\left(n+\frac{1}{2}\right)\left(e^{2} / h\right)$, which (after accounting for the fourfold degeneracy of the Dirac fermion) has been seen in graphene $[20,21]$. FQH plateaux have also been observed in graphene [22,23]; there is intriguing evidence that the FQH effect may exist on the surface of TIs [24]. In contrast to the nonrelativistic case, for Dirac fermions, particle-hole symmetry is a good symmetry even with Landau level mixing. It is not obvious that the flux attachment procedure can be carried out for Dirac fermions; the usual workaround is to work in the limit of zero Landau-level mixing where the projected Hamiltonian is identical to the nonrelativistic one [25]. This method explicitly breaks particle-hole symmetry and, furthermore, does not work at finite Landau level mixing.

In this paper, we propose an explicitly particle-hole symmetric effective theory describing the low-energy dynamics of the Fermi liquid state of a half-filled Landau level. This theory is constructed to respect all discrete symmetries and to satisfy phenomenological constraints, in particular, the existence of the two Jain sequences below and above $\nu=\frac{1}{2}$. Our proposal is similar to the fermionic Chern-Simons (HLR) theory in that the elementary degree of freedom is a fermion; however, it differs from it in several ways: (i) The fermion is, by nature, a Dirac fermion.

(ii) The fermion is its own particle-hole conjugate.

(iii) The fermion has no Chern-Simons interactions. This is an important point as the Chern-Simons term is not consistent with particle-hole symmetry [26].

As per point (i), one may wonder if there is any difference between the Fermi liquid of Dirac fermions and that of nonrelativistic fermions: Both are characterized by a linear dispersion relation of quasiparticles near the Fermi surface. The difference is in the Berry phase when the quasiparticle is moved around the Fermi surface (a circle in 2D), which is $\pm \pi$ in the case of a Dirac fermion. The importance of the Berry phase as a property of the Landau fermion quasiparticle was emphasized by Haldane [28].

The Berry phase of $\pi$ offers a resolution to the puzzle of the anomalous Hall conductivity of the CFs. As shown by Haldane [28], the unquantized part of the anomalous Hall conductivity of the CF Fermi liquid is equal to the global Berry phase $\gamma$, which the quasiparticle receives when it moves around the Fermi disk,

$$
\sigma_{x y}^{\mathrm{CF}}=\left(\frac{\gamma}{2 \pi}+n\right) \frac{e^{2}}{h}, \quad n \in \mathbb{Z} .
$$

When $\gamma= \pm \pi$, this equation is consistent with $\sigma_{x y}^{\mathrm{CF}}=-\frac{1}{2}$. The resolution to the puzzle is not the noncommutativity of the $m \rightarrow 0$ limit and the clean limit; rather, it is in an ingredient of the Fermi liquid theory missing in all treatments of the CFs so far: the global Berry phase.

Beside these differences, there are also many similarities between the picture proposed here and the standard CF picture. In particular, the fermion quasiparticle is electrically neutral. The Jain-sequence states near half filling are mapped to IQH states of the CFs; however, in our theory, particle-hole conjugate states map to IQH states with the same half-integer filling factors.

We show that the theory suggests the existence of a particle-hole symmetric gapped state at $\nu=\frac{1}{2}$, distinct from the Pfaffian and anti-Pfaffian states.

The structure of the paper is as follows. In Sec. II, we start by writing down a model consisting of a single twocomponent Dirac fermion, localized on a $(2+1) D$ "brane," coupled to electromagnetism in four dimensions. This model does not contain complications specific for graphene or the surface state of TIs (the fourfold degeneracy in graphene, or the Zeeman coupling in the case of TIs) and should realize a Fermi-liquid state in a finite magnetic field. We show that the electromagnetic response in this model is related directly to that of the nonrelativistic electrons on the LLL. We use the model to discuss discrete symmetries, which are also shared by the nonrelativistic model in the LLL limit, emphasizing that there are two independent discrete symmetries in the finite magnetic field at charge neutrality: $\mathcal{C P}$ and $\mathcal{P} \mathcal{T}$. We then put forward, in Sec. III, our proposal for the low-energy effective field theory and 
discuss physical implications. The possible connection to the conventional fermionic Chern-Simons theory is discussed in Sec. IV. Finally, Sec. V contains concluding remarks.

\section{A RELATIVISTIC MODEL REALIZING THE $\nu=\frac{1}{2}$ FQH STATE}

To be more specific, we discuss a theory of a massless fermion localized on a $(2+1)$-dimensional brane placed at $z=0$, interacting through a $\mathrm{U}(1)$ gauge field in the $(3+1)$ dimensional bulk,

$$
S=\int d^{3} x i \bar{\Psi} \gamma^{\mu}\left(\partial_{\mu}-i A_{\mu}\right) \Psi-\frac{1}{4 e^{2}} \int d^{4} x F_{\mu \nu}^{2},
$$

where $\Psi$ is a two-component spinor. We choose the following representation for the gamma matrices:

$$
\gamma^{0}=\sigma^{3}, \quad \gamma^{1}=i \sigma^{2}, \quad \gamma^{2}=-i \sigma^{1} .
$$

One can think of $\Psi$ as the fermion zero mode localized on a domain wall. In the condensed-matter language, $\Psi$ is the surface mode of a 3D TI. The theory has one dimensionless coupling constant $e$. In contrast to the usual QED, $e$ does not run since it determines the strength of the electromagnetic interactions infinitely far away from the brane, where there is no matter that would renormalize it. We are mostly interested in the weak coupling regime where $e^{2} \ll 1$, but most of our statements should be valid up to some finite value of $e^{2}$.

Our task is to understand the ground state of the system in the finite magnetic field $B=F_{x y}$ and its excitations. This problem is nonperturbative even at $e^{2} \ll 1$ since it maps to a FQH problem. To see this, assume at first that $e^{2}=0$, and recall that the Landau levels of the Dirac Hamiltonian are $E= \pm \sqrt{2 n B}$. In the ground state, the states with negative energy are filled and those with positive energy are empty, but the noninteracting Hamiltonian gives us no prescription for the $n=0$ LL (the zeroth LL), whose states have exact zero energy. The true ground state is only singled out when interaction is turned on. It is worth noting that the energy scale induced by the interaction is $e^{2} \sqrt{B}$, while the spacing between LLs is $\sqrt{B}$.

To see that the problem maps to the $\nu=\frac{1}{2} \mathrm{QH}$ problem, we notice that, because of particle-hole symmetry, at zero chemical potential, the zeroth LL must be half full (or half empty). At small $e^{2}$, all essential physics occurs on the zeroth LL, and the Hamiltonian projected to this LL is the same as the usual projected QH Hamiltonian since the Dirac orbitals on the zeroth LL are the same (neglecting one component of the Dirac spinor which vanishes for these states) as the Landau orbitals of a nonrelativistic particle [see Eq. (27) below].

\section{A. Equivalence between Dirac fermions and nonrelativistic fermions on the LLL}

\section{Universality of the LLL limit}

We have argued that the problem of finding the ground state of a Dirac fermion in a magnetic field, in the weak coupling regime $e^{2} \ll 1$, is equivalent to finding the ground state of a nonrelativistic fermion in the LLL limit $m \rightarrow 0$. In this subsection, we show that the equivalence can be extended further: The full electromagnetic response of one theory can be obtained from that of another theory. It is obvious that the density response to an external potential is the same in the two theories. The relationship between the currents in the two theories is, however, slightly more complicated.

To establish the connection, we recall a recent procedure to derive the expression for the current in the $m \rightarrow 0$ limit of a nonrelativistic theory [29]. We start from the Lagrangian

$$
\mathcal{L}=i \psi^{\dagger} D_{t} \psi-\frac{1}{2 m} D_{i} \psi^{\dagger} D_{i} \psi+\mathcal{L}_{\text {int }} .
$$

In later formulas, we drop the interaction term $\mathcal{L}_{\text {int }}$, always implicitly implying it. We now add to the Lagrangian a magnetic-moment term, giving the particle a gyromagnetic factor $g=2$ (and assuming the system is fully spin polarized),

$$
\mathcal{L}_{g=2}=i \psi^{\dagger} D_{t} \psi-\frac{1}{2 m} D_{i} \psi^{\dagger} D_{i} \psi+\frac{B}{2 m} \psi^{\dagger} \psi .
$$

For a constant magnetic field, the added term is proportional to the total number of particles, which commutes with the Hamiltonian and does not alter the dynamics. However, the expression for the electromagnetic current $j_{i}=\delta S / \delta A_{i}$ has changed. Denoting the current corresponding to Eq. (4) by $j_{g=0}^{i}$ and to Eq. (5) by $j_{g=2}^{i}$, we find

$$
j_{g=0}^{i}=j_{g=2}^{i}-\frac{1}{2 m} \epsilon^{i j} \partial_{j} \rho .
$$

The two currents differ only by a solenoidal term, and if we know the density response, we can get one current from the other. As we will see later, $j_{g=2}^{i}$ is finite in the limit $m \rightarrow 0$, but $j_{g=0}^{i}$ is, in general, divergent. In the subsequent discussion, by the electromagnetic current we have in mind $j_{g=2}^{i}$ by default. Note that for linear response at $q=0$ (but any frequency), the two currents coincide.

Ignoring a total derivative, the Lagrangian (5) can be rewritten as

$$
\mathcal{L}=i \psi^{\dagger} D_{t} \psi-\frac{1}{2 m}\left(D_{x}-i D_{y}\right) \psi^{\dagger}\left(D_{x}+i D_{y}\right) \psi .
$$

Introducing auxiliary fields $\chi$ and $\chi^{\dagger}$, we can recast the Lagrangian in the form 


$$
\begin{aligned}
\mathcal{L}= & i \psi^{\dagger} D_{t} \psi+i \psi^{\dagger}\left(D_{x}-i D_{y}\right) \chi+i \chi^{\dagger}\left(D_{x}+i D_{y}\right) \psi \\
& +2 m \chi^{\dagger} \chi .
\end{aligned}
$$

Now, taking the $m \rightarrow 0$ limit, the Lagrangian becomes

$$
\mathcal{L}=i \psi^{\dagger} D_{t} \psi+i \psi^{\dagger}\left(D_{x}-i D_{y}\right) \chi+i \chi^{\dagger}\left(D_{x}+i D_{y}\right) \psi .
$$

The last form of the Lagrangian makes it clear that the limit $m \rightarrow 0$ is finite. In fact, Eq. (9) can be thought of as a Lagrangian formulation of the problem on the lowest Landau level. The variables $\chi$ and $\chi^{\dagger}$ are Lagrange multipliers enforcing the constraints

$$
\left(D_{x}+i D_{y}\right) \psi=0, \quad\left(D_{x}-i D_{y}\right) \psi^{\dagger}=0,
$$

which is nothing but the LLL constraint. For example, in the symmetric gauge $A_{x}=-\frac{1}{2} B y, A_{y}=\frac{1}{2} B x$, the constraints imply that $\psi$ is a linear combination of Landau's orbitals $z^{n} e^{-B|z|^{2} / 4}, n=0,1,2 \ldots$ Equation (9) implies that the physics on the LLL is universal, i.e., independent of how the LLL limit is approached. In Ref. [29], it was shown that the current computed from Eq. (9) coincides with that found in Ref. [30]. This current differs from the one obtained in Ref. [31] by a solenoidal term, which is not surprising since Ref. [31] assumes a zero geomagnetic factor and should gives $j_{g=0}^{i}$ instead of $j_{g=2}^{i}$.

Let us now consider the Dirac field theory. Denoting the two components of the Dirac spinor as

$$
\psi=\left(\begin{array}{l}
\psi \\
\chi
\end{array}\right),
$$

the Dirac action can be written as

$$
\begin{aligned}
\mathcal{L}= & i \psi^{\dagger} D_{t} \psi+i \chi^{\dagger} D_{t} \chi+i \psi^{\dagger}\left(D_{x}-i D_{y}\right) \chi \\
& +i \chi^{\dagger}\left(D_{x}+i D_{y}\right) \psi .
\end{aligned}
$$

If one concentrates on states on the zeroth Landau level, the Dirac spinor will have $\chi$ much smaller than $\psi$. In the Lagrangian, the term $\chi^{\dagger} \partial_{t} \chi$ is quadratic in $\chi$ and can be neglected compared to other terms. Terms linear in $\chi$ will still have to be kept since the time derivative $D_{t}$ is of the same order of smallness as $\chi$. Thus, the Lagrangian becomes exactly Eq. (9).

\section{Relationship between currents in relativistic and nonrelativistic theories}

Although the expressions of the current coming from the modes on the LLL in the relativistic and nonrelativistic theories are the same, the physics of the relativistic model is slightly different from that of the nonrelativistic one by the presence of a Dirac sea of Landau levels with negative energies. The states in the Dirac sea contribute a uniform charge density $-B / 4 \pi$; thus,

$$
\rho_{\mathrm{D}}=\rho_{\mathrm{NR}}-\frac{B}{4 \pi}
$$

where the subscripts "D" and "NR" refer to "Dirac" and "nonrelativistic," respectively. To find the contribution of the sea to the current, we notice that the electrons in the Dirac sea are subject to an additional scalar potential created by the electrons on the zeroth Landau level,

$$
V_{\mathrm{eff}}(\mathbf{x})=\int d \mathbf{y} V(\mathbf{x}-\mathbf{y}) \rho(\mathbf{x}) \equiv(V \cdot \rho)(\mathbf{x}),
$$

where $V(\mathbf{x}-\mathbf{y})$ is the interaction potential between electrons (i.e., the Coulomb potential). The response of the Dirac sea to this scalar potential gives an extra contribution to the current, leading to the following relationship between the currents:

$$
j_{\mathrm{D}}^{i}=j_{\mathrm{NR}}^{i}+\frac{1}{4 \pi} \epsilon^{i j} \partial_{j}(V \cdot \rho) .
$$

The two formulas can be summarized as the following relationship between the logarithms of the partition functions $(W=-i \ln Z)$ in the two theories, valid for any external $A_{0}$ and to linear order in perturbations of $A_{i}$ around the background,

$$
W_{\mathrm{D}}\left[A_{0}, A_{i}\right]=-\frac{A_{0} B}{4 \pi}+W_{\mathrm{NR}}\left[A_{0}+\frac{V \cdot B}{4 \pi}, A_{i}\right] .
$$

Differentiating with respect to $A_{0}$ and $A_{i}$ we recover Eqs. (13) and (15).

The formulas are written in the long-wavelength limit of external probes. This is sufficient for the purpose of the rest of the paper. For completeness, we mention that there are corrections to the formulas from two sources: (i) the wave-number dependence of the Hall conductivity of the filled levels, and (ii) the exchange interactions between the electrons in the Dirac sea and those on the zeroth Landau level [only the Hartree interaction has been taken into account in Eq. (14)]. Both effects are small in the longwavelength limit for the potential $V$ sufficiently long ranged so that its Fourier transform $V(q)$ diverges at $q \rightarrow 0$ (as the Coulomb potential).

\section{Relativistic convention}

The discussion above shows that there is a direct relationship between physical observables in the cases of Dirac and nonrelativistic fermions in the LLL limit. Here, we collect formulas that relate quantities in the two theories, which will be useful in our further discussion. 
We define the filling factor as the ratio

$$
\nu=\frac{\rho}{B / 2 \pi},
$$

where $\rho$ is the charge density. According to Eq. (13), the relationship between the relativistic and nonrelativistic filling factors is

$$
\nu=\nu_{\mathrm{NR}}-\frac{1}{2},
$$

where we have dropped the index " $\mathrm{D}$ " on the left-hand side. In the relativistic theory, $\nu=0$ occurs at zero chemical potential (the charge neutrality point), where the $n=0$ Landau level is half filled. The latter corresponds to $\nu_{\mathrm{NR}}=1 / 2$.

Similarly, one can derive a relationship between the conductivities in the relativistic and nonrelativistic theories from Eq. (15). At zero wave number (but any frequency), the spatial derivative term on the right-hand side of Eq. (15) vanishes, giving

$$
\begin{aligned}
& \sigma_{x x}(\omega)=\sigma_{x x}^{\mathrm{NR}}(\omega), \\
& \sigma_{x y}(\omega)=\sigma_{x y}^{\mathrm{NR}}(\omega)-\frac{1}{2} .
\end{aligned}
$$

We have measured the conductivities in units of $e^{2} / h$. One can interpret Eq. (20) as the statement that the Hall conductivity of the Dirac fermion consists of a contribution from the zeroth Landau level, equal to $\sigma_{x y}^{\mathrm{NR}}$, and the contribution from the filled negative-energy Landau levels, equal to $-\frac{1}{2}$. Note that these formulas are valid also in the presence of impurities.

Next we consider the shift. In the relativistic context, instead of the shift, it is convenient to parametrize a given gapped quantum Hall state by a parameter $\kappa$ giving the offset between the total charge $N_{e}$ and the total magnetic flux in the unit of flux quantum $N_{\phi}$, on a sphere [32]:

$$
N_{e}=\nu N_{\phi}+\kappa .
$$

To compare $\kappa$ with the shift of the corresponding QH state on the LLL, one has to take into account two facts: There is a $\frac{1}{2}$ offset between the definitions of the relativistic and nonrelativistic filling factors, $\nu=\nu_{\mathrm{NR}}-\frac{1}{2}$, and that Dirac particle has a direct coupling to the spin connection. As a result, the connection between $\kappa$ and the shift $\mathcal{S}$ is [32]

$$
\kappa=\nu_{\mathrm{NR}}(\mathcal{S}-1) .
$$

In particular, if two states, "1" and "2," are particle-hole conjugates of each other, then $\nu_{1}^{\mathrm{NR}}+\nu_{2}^{\mathrm{NR}}=\nu_{1}^{\mathrm{NR}} \mathcal{S}_{1}+$ $\nu_{2}^{\mathrm{NR}} \mathcal{S}_{2}=1$, which in the relativistic notations become simply

$$
\nu_{1}+\nu_{2}=\kappa_{1}+\kappa_{2}=0 .
$$

Another quantity, which will not be discussed in this paper, is the chiral central charge $c$. The relationship between the relativistic and nonrelativistic convention for this charge is $c=c_{\mathrm{NR}}-\frac{1}{2}$. As for $\nu$ and $\kappa$, particle-hole conjugation flips the sign of $c$.

\section{B. Discrete symmetries}

We recall the theory (2) has the following symmetries [33]:

(i) Charge conjugation,

$$
\begin{aligned}
\mathcal{C} A_{\mu} \mathcal{C}^{-1} & =-A_{\mu}, \\
\mathcal{C} \Psi \mathcal{C}^{-1} & =\sigma^{1} \Psi^{*}
\end{aligned}
$$

(ii) Spatial parity $\mathbf{x}=(x, y) \rightarrow \mathbf{x}^{\prime}=(x,-y)$,

$$
\begin{aligned}
& \mathcal{P} A_{0}(t, \mathbf{x}) \mathcal{P}^{-1}=A_{0}\left(t, \mathbf{x}^{\prime}\right), \\
& \mathcal{P} A_{1}(t, \mathbf{x}) \mathcal{P}^{-1}=A_{1}\left(t, \mathbf{x}^{\prime}\right), \\
& \mathcal{P} A_{2}(t, \mathbf{x}) \mathcal{P}^{-1}=-A_{2}\left(t, \mathbf{x}^{\prime}\right), \\
& \mathcal{P} \Psi(t, \mathbf{x}) \mathcal{P}^{-1}=\sigma^{1} \Psi\left(t, \mathbf{x}^{\prime}\right) .
\end{aligned}
$$

(iii) Time reversal $t \rightarrow-t$,

$$
\begin{aligned}
& \mathcal{T} A_{0}(t, \mathbf{x}) \mathcal{T}^{-1}=A_{0}(-t, \mathbf{x}), \\
& \mathcal{T} A_{i}(t, \mathbf{x}) \mathcal{T}^{-1}=-A_{i}(-t, \mathbf{x}), \\
& \mathcal{T} \Psi(t, \mathbf{x}) \mathcal{T}^{-1}=-i \sigma^{2} \Psi(-t, \mathbf{x}) .
\end{aligned}
$$

$\mathcal{T}$ is an anti-unitary operator $\left(\mathcal{T} i \mathcal{T}^{-1}=-i\right)$. These individual symmetries are broken by the magnetic field $B$ which changes sign under each of $\mathcal{C}, \mathcal{P}$, and $\mathcal{T}$. However, $\mathcal{C P}, \mathcal{C} \mathcal{T}$, and $\mathcal{P} \mathcal{T}$ leave the magnetic field unchanged and hence remain the discrete symmetries of the Dirac fermion in a magnetic field [32]. We lose one symmetry because only two out of these three are independent, e.g., $\mathcal{C T} \sim$ $(\mathcal{C P})(\mathcal{P} \mathcal{T})$. The chemical potential further breaks $\mathcal{C P}$ and $\mathcal{C} \mathcal{T}$, leaving $\mathcal{P} \mathcal{T}$ as the only symmetry of the Dirac fermion in a magnetic field at nonzero chemical potential. As we are interested mostly in the charge neutrality point, $\mathcal{C P}$ and $\mathcal{C T}$ will be exploited as symmetries [34].

When $e^{2} \ll 1$, all interesting physics occurs on the $n=0$ Landau level, and the $\mathcal{C P}, \mathcal{C} \mathcal{T}$, and $\mathcal{P} \mathcal{T}$ operators map to symmetries of the LLL Hamiltonian. Let us choose the symmetric gauge, where the single-particle orbitals on 
the LLL are $z^{m} e^{-|z|^{2} / 4}$. The field $\Psi$ projected to the LLL can be written as

$$
\Psi=\sum_{m}\left(\begin{array}{c}
A_{m} z^{m} e^{-B|z|^{2} / 4} \\
0
\end{array}\right) c_{m},
$$

where $A_{m}$ is a normalization coefficient and $c_{m}$ is the operator annihilating a fermion on the orbital $\mathrm{m}$. Then, from Eqs. (24b), (25d), and (26c)), we find the action of the symmetries on $c_{m}$,

$$
\begin{aligned}
& \mathcal{C} \mathcal{P} c_{m}(\mathcal{C P})^{-1}=c_{m}^{\dagger}, \\
& \mathcal{P} \mathcal{T} c_{m}(\mathcal{P} \mathcal{T})^{-1}=c_{m}, \\
& \mathcal{C} \mathcal{T} c_{m}(\mathcal{C T})^{-1}=c_{m}^{\dagger} .
\end{aligned}
$$

Note that $\mathcal{C} \mathcal{T}$ is different from $\mathcal{C P}$ : The former is an antiunitary transformation, while the latter is unitary. In the quantum Hall literature, by particle-hole symmetry, one normally has in mind $\mathcal{C} \mathcal{T}$. Likewise, $\mathcal{P} \mathcal{T}$ is not an identity operator: It replaces the wave function of a given state, in the basis obtained by acting $c_{m}^{\dagger}$ 's on the vacuum, with its complex conjugate. More precisely, if $|f\rangle$ is the following quantum $N$-body state on the LLL,

$$
|f\rangle=\sum_{\left\{n_{i}\right\}} f_{n_{1} n_{2} \ldots n_{N}} c_{n_{1}}^{\dagger} c_{n_{2}}^{\dagger} \cdots c_{n_{N}}^{\dagger}+|0\rangle,
$$

then

$$
\mathcal{P} \mathcal{T}|f\rangle=\sum_{\left\{n_{i}\right\}} f_{n_{1} n_{2} \ldots n_{N}}^{*} c_{n_{1}}^{\dagger} c_{n_{2}}^{\dagger} \cdots c_{n_{N}}^{\dagger}+|0\rangle .
$$

Note that the Laughlin states [2] and the Moore-Read states [10] are invariant under $\mathcal{P} \mathcal{T}$ : In both cases, the coefficients appearing in the holomorphic polynomial in the wave function are all real. We are unaware of any trial wave function that is not invariant under $\mathcal{P} \mathcal{T}$. Assuming $\mathcal{P} \mathcal{T}$ symmetry, $\mathcal{C P}$ is equivalent to $\mathcal{C T}$; thus, we will sometimes call $\mathcal{C P}$ the particle-hole symmetry, although in the literature the latter usually corresponds to $\mathcal{C} \mathcal{T}$.

\section{Consequences of discrete symmetries for linear response}

Consider the linear response of a $\mathrm{QH}$ system to a small perturbation of the electromagnetic potential: $j^{\mu}(q)=\Pi^{\mu \nu}(q) A_{\nu}(q), \quad q_{\mu} \Pi^{\mu \nu}=0$. Assuming rotational invariance, there are three independent components of the polarization tensor $\Pi^{\mu \nu}$,

$$
\Pi^{i j}=\frac{q^{i} q^{j}}{q^{2}} \Pi_{\mathrm{L}}+\left(\delta^{i j}-\frac{q^{i} q^{j}}{q^{2}}\right) \Pi_{\mathrm{T}}+\epsilon^{i j} \Pi_{\mathrm{H}}
$$

The Hall conductivity $\sigma_{x y}$ is related to $\Pi_{\mathrm{H}}$ by $\Pi_{\mathrm{H}}=$ $(i / 2 \pi) \omega \sigma_{x y}$. At nonzero chemical potential, $\mathcal{P} \mathcal{T}$ is the only symmetry, and we get the constraints $\Pi_{\mathrm{L}, \mathrm{T}}(-\omega, q)=$ $\Pi_{\mathrm{L}, \mathrm{T}}^{*}(\omega, q)$ and $\sigma_{x y}(-\omega, q)=\sigma_{x y}^{*}(\omega, q)$, but at charge neutrality, $\mathcal{C P}$ implies that the Hall conductivity vanishes,

$$
\sigma_{x y}(\omega, q)=0,
$$

at any value of $\omega$ and $q$. This is true not only for clean systems but also in the presence of impurities, provided that the latter do not statistically break the particle-hole symmetry.

For nonrelativistic fermions on the LLL, we can use Eq. (20) to find, for $q=0$,

$$
\sigma_{x y}^{\mathrm{NR}}(\omega)=\frac{1}{2},
$$

generalizing a result derived in Ref. [15] to nonzero frequencies. For $q \neq 0$, this relationship is replaced by a linear constraint on $\sigma_{x y}$ and $\Pi_{\mathrm{L}}$ [36].

An explicitly particle-hole symmetric theory of the halffilled state should imply Eq. (34) automatically. We now construct such a theory.

\section{PROPOSAL FOR THE LOW-ENERGY EFFECTIVE FIELD THEORY}

Lacking a better way, we are going to simply guess the form of the low-energy effective theory. We start by stating a few requirements that our theory should satisfy:

(i) The theory should be invariant under $\mathcal{C P}$ and $\mathcal{P} \mathcal{T}$.

(ii) At charge neutrality and nonzero magnetic field, the ground state should be a Fermi liquid.

(iii) The theory should explain the Jain sequences.

The requirement (ii) is particularly nontrivial, as Luttinger's theorem requires the volume of the Fermi sphere to be proportional to the density of some charge, but at charge neutrality, the electromagnetic charge density is equal to zero.

It turns out that the three requirements above are satisfied by the following action, which we propose as the lowenergy effective theory:

$$
\begin{aligned}
S_{\text {eff }}= & \int d^{3} x\left(i \bar{\psi} \gamma^{\mu}\left(\partial_{\mu}+2 i a_{\mu}\right) \psi+\frac{1}{2 \pi} \epsilon^{\mu \nu \lambda} A_{\mu} \partial_{\nu} a_{\lambda}\right) \\
& -\frac{1}{4 e^{2}} \int d^{4} x F_{\mu \nu}^{2}+\cdots .
\end{aligned}
$$

Here, $\psi$ is a Dirac field describing the fermionic quasiparticle, $a_{\mu}$ is an emergent gauge field, and ... stands for other terms, including a possible Maxwell kinetic term for $a_{\mu}$ and interaction terms. The quasiparticle $\psi$, which will also be called the Dirac CF, has quantum numbers different from the electron: It is electrically neutral and 
carries charge with respect to the emergent gauge field $a_{\mu}$. Note that in the HLR theory, the CF is also electrically neutral, a point which has been emphasized by Read [37,38].

Going from the microscopic action (2) to Eq. (36), it looks as if the electron $\Psi$ has been stripped off of its charge, whose dynamics is now governed by a dual gauge field $a_{\mu}$. Interestingly, a similar phenomenon has recently been suggested to occur in the "composite Dirac liquid" state [39] - a possible parent to several gapped topological phases of the strongly interacting surface of 3D TI [40-43]. As in our proposed effective theory, the only low-energy mode of the composite Dirac liquid is a neutral Dirac fermion. While it is certain that the compositefermion liquid phase cannot occur in the theory (2) in the zero magnetic field in the limit $e^{2} \ll 1$, what we are suggesting is that as soon as one turns on a magnetic field, the most convenient representation of the low-energy dynamics is not in terms of the original fermion but in terms of a composite Dirac fermion of the type considered in Ref. [39].

In Eq. (36), we normalize the field $a_{\mu}$ so that a $2 \pi$ magnetic flux of $a$ carries a unit electric charge [see Eq. (41) below]. With this normalization, the charge of the $\psi$ field is fixed to 2 . As we will see below, this value of the charge is required for the theory to be consistent with Jain's sequences, the Fermi momentum of the half-filled state, and the $e / 4$ charged excitation in the Pfaffian state.

\section{A. Discrete symmetries of the effective field theory}

It is easy to check that the Lagrangian (36) exhibits the full set of discrete symmetries of the original theory (2), including

(i) charge conjugation,

$$
\begin{aligned}
\mathcal{C} a_{\mu}(t, \mathbf{x}) \mathcal{C}^{-1} & =-a_{\mu}(t, \mathbf{x}), \\
\mathcal{C} \psi(t, \mathbf{x}) \mathcal{C}^{-1} & =\sigma^{1} \psi^{*}(t, \mathbf{x}) ;
\end{aligned}
$$

(ii) spatial parity,

$$
\begin{aligned}
& \mathcal{P} a_{0}(t, \mathbf{x}) P^{-1}=-a_{0}\left(t, \mathbf{x}^{\prime}\right), \\
& \mathcal{P} a_{1}(t, \mathbf{x}) P^{-1}=-a_{1}\left(t, \mathbf{x}^{\prime}\right), \\
& \mathcal{P} a_{2}(t, \mathbf{x}) P^{-1}=a_{2}\left(t, \mathbf{x}^{\prime}\right), \\
& \mathcal{P} \psi(t, \mathbf{x}) P^{-1}=\psi^{*}\left(t, \mathbf{x}^{\prime}\right) ;
\end{aligned}
$$

(iii) time reversal,

$$
\mathcal{T} a_{0}(t, \mathbf{x}) T^{-1}=-a_{0}(-t, \mathbf{x}),
$$

$$
\begin{aligned}
& \mathcal{T} a_{i}(t, \mathbf{x}) T^{-1}=a_{i}(-t, \mathbf{x}), \\
& \mathcal{T} \psi(t, \mathbf{x}) T^{-1}=\sigma^{3} \psi^{*}(-t, \mathbf{x}) .
\end{aligned}
$$

[We omit the transformation laws for $A_{\mu}$ which are the same as in Eqs. (24), (25), and (26)]. In particular, our effective theory is invariant under $\mathcal{C P}$ and $\mathcal{P} \mathcal{T}$. We note that the parity anomaly is avoided in this theory by the charge 2 of the CF. Note that the fermion mass term $m \bar{\psi} \psi$ and the Chern-Simons term for $a_{\mu}$ are allowed by $\mathcal{P} \mathcal{T}$ but forbidden by $\mathcal{C P}$ or $\mathcal{C}$. But we expect the terms not forbidden by symmetries to appear in the "..." in Eq. (36).

It is instructive to write down the transformation law for $\psi$ under $\mathcal{C P}, \mathcal{C} \mathcal{T}$,

$$
\begin{gathered}
\mathcal{C P} \psi(t, \mathbf{x})(\mathcal{C P})^{-1}=\sigma^{1} \psi(t,-\mathbf{x}), \\
\mathcal{C} \mathcal{T} \psi(t, \mathbf{x})(\mathcal{C} \mathcal{T})^{-1}=-i \sigma^{2} \psi(-t, \mathbf{x}), \\
\mathcal{P} \mathcal{T} \psi(t, \mathbf{x})(\mathcal{P} \mathcal{T})^{-1}=\sigma^{3} \psi(-t,-\mathbf{x}) .
\end{gathered}
$$

Note that under particle-hole symmetries $(\mathcal{C P}$ and $\mathcal{C T}), \psi$ does not transform into the complex conjugated field $\psi^{*}$ but remains $\psi$. In fact, the transformation laws of $\psi$ under $\mathcal{C P}$ and $\mathcal{C T}$ are the same as those of the original electrons $\Psi$ under $\mathcal{P}$ and $\mathcal{T}$ [Eqs. (25d) and (26c)]. Thus, particle-hole symmetry does not transform the composite fermion $\psi$ into its antiparticle but leaves it as a particle. This result can also be seen from the fact that $a_{0}$, which is proportional to the chemical potential of the $\mathrm{CFs}$, does not change sign under $\mathcal{C P}$ and $\mathcal{C T}$.

\section{B. Fermi liquid and Jain sequences}

To establish the nature of the $\mathrm{QH}$ state at charge neutrality, we first note that the electromagnetic current defined by Eq. (36) is

$$
j^{\mu}=\frac{1}{2 \pi} \epsilon^{\mu \nu \lambda} \partial_{\nu} a_{\lambda}
$$

In particular, charge density is related to the emergent magnetic field: $b=2 \pi \rho$. At charge neutrality, $\rho=0$, and therefore the fermionic quasiparticles feel a zero magnetic field.

On the other hand, differentiating the action with respect to $a_{0}$, one finds a relationship between the density of the Dirac CF and the magnetic field,

$$
\tilde{\rho} \equiv\left\langle\bar{\psi} \gamma^{0} \psi\right\rangle=\frac{B}{4 \pi} .
$$

Therefore, in a nonzero magnetic field, the CFs have a finite density and live in a zero magnetic field. 
Assuming no Cooper instability (a possibility that we will consider later), the ground state is then a Fermi liquid of the CFs, and Luttinger's theorem fixes the Fermi momentum to the inverse of the magnetic length. The value of the Fermi momentum is the same as the value in the standard HLR theory [5]. Note that the Fermi velocity $v_{F}$ of the $\mathrm{CF}$ is, in general, different from 1; at weak coupling, $v_{F}$ should scale with the interaction energy: $v_{F} \sim e^{2}$. The renormalization of the Fermi velocity is related to Landau's Fermi liquid parameters [44]. The effective theory can be trusted to describe the dynamics of the fermions near the Fermi surface but not far away from it. In principle, one should be able to reformulate the theory completely in terms of the degree of freedom near the Fermi surface $[45,46]$, but since preserving the information about the Berry phase (which will be important when we discuss the Jain sequences) is quite nontrivial, we will not try to do so.

The fermion quasiparticle is the $\mathcal{C P}$ conjugate of itself. To see it, we note that $\mathcal{C P} \psi(\mathcal{C P})=\sigma^{1} \psi$. Consider a particle moving with momentum $\mathbf{p}=(p, 0)$, choosing the $\mathbf{p}$ to be invariant under $y \rightarrow-y$. Its wave function is an eigenvector of the Dirac Hamiltonian $\boldsymbol{\sigma} \cdot \mathbf{p}=\sigma^{1} p$, which is $(1,0)^{T}$. This wave function is invariant under $\mathcal{C P}$.

Away from charge neutrality, the fermion quasiparticles feel an effective magnetic field equal to

$$
\tilde{B}=-2 \nabla \times a=-4 \pi \rho .
$$

Equations (42) and (43) tell us that from the point of view of the composite fermions, the notions of density and magnetic field get swapped, similarly to what happens under particle-vortex duality for bosons [47]. As a result, the filling factor of the original electrons $\nu=2 \pi \rho / B$ and the effective filling factor of the fermion quasiparticles $\tilde{\nu}=2 \pi \tilde{\rho} / \tilde{B}$ are inversely proportional to each other,

$$
2 \nu=-\frac{1}{2 \tilde{\nu}} .
$$

This means the Jain-sequence state with filling factor $\nu=n /(2 n+1)-\frac{1}{2}=-1 /[2(2 n+1)]$ maps to an IQH state of the fermion quasiparticle with $\tilde{\nu}=n+\frac{1}{2}$. Note that the filling factor $\nu=(n+1) /(2 n+1)-\frac{1}{2}$ maps to $\tilde{\nu}=-\left(n+\frac{1}{2}\right)$, making explicit the fact that the two states are particle-hole conjugates of each other.

\section{Shift of states on the Jain sequences}

A nontrivial check for the proposed effective field theory is the computation of the shift [48] of the states on the Jain sequences on a sphere. Consider the $\nu_{\mathrm{NR}}=$ $(n+1) /(2 n+1)$ state. The fermions $\psi$ are effectively in the magnetic field of $2 b$ in the IQH state with $\nu_{\text {eff }}=-\left(n+\frac{1}{2}\right)$. From solving the Dirac equation on a sphere, the degeneracy of the $n$th Landau level is $N_{\phi}+2|n|$. Therefore,

$$
\int d \mathbf{x}\left\langle\psi^{\dagger} \psi\right\rangle=\left(n+\frac{1}{2}\right) \int d \mathbf{x} \frac{2 b}{2 \pi}+n(n+1) .
$$

But $\rho=b / 2 \pi$ and $\left\langle\psi^{\dagger} \psi\right\rangle=B / 4 \pi$; therefore, we have

$$
\frac{N_{\phi}}{2}=(2 n+1) N_{e}+n(n+1) .
$$

This means [see Eq. (21)]

$$
\nu=\frac{1}{2(2 n+1)}, \quad \kappa=-\frac{n(n+1)}{2 n+1},
$$

so $\nu_{\mathrm{NR}}=(n+1) /(2 n+1)$ and, from Eq. (22), $\mathcal{S}=$ $-n+1$. The shift of the $\nu_{\mathrm{NR}}=n /(2 n+1)$ can be computed similarly to be $\mathcal{S}=n+2$. These values of the shift coincide with what is known about these states [48].

\section{Pfaffian, anti-Pfaffian, and a particle-hole symmetric non-Abelian state}

We can construct various gapped states by letting $\psi$ form a BCS pair. Because the Dirac fermion has an additional Berry phase around the origin in momentum space, pairing occurs in channels with even angular momentum.

Consider first $s$-wave pairing, with the order parameter being $\psi^{T} \sigma_{2} \psi$. It is easy to see that the order parameter is invariant under $\mathcal{C P}, \mathcal{C} \mathcal{T}$, and $\mathcal{P} \mathcal{T}$ symmetries (combined with phase rotations of $\psi$ when required).

Since this is a particle-hole symmetric state, it cannot be the Pfaffian or anti-Pfaffian state. In the conventional composite fermion picture, this state can be understood as a state where the composite fermions form Cooper pairs in the $p_{x}-i p_{y}$ channel with the orbital angular momentum opposite to the Moore-Read state. While the conventional picture does not immediately tell us so, we find here that this state coincides with its particle-hole conjugate. In particular, for this state, $\kappa=0$, corresponding to the shift $\mathcal{S}=1$. Because of the condensation of Cooper pairs that carry charge 4 under $a_{\mu}$, the vortices in the condensate correspond to quasiparticles with charge $\pm 1 / 4$. The vortex has fermionic zero mode and should have non-Abelian statistics. This state is a particle-hole symmetric Pfaffianlike state, which we will term the PH-Pfaffian. In fact, its construction parallels that of the time-reversal-invariant T-Pfaffian state on the surface of TIs [41,42].

The Pfaffian and anti-Pfaffian states correspond to pairing of $\psi$ in the $\ell= \pm 2$ channels. The vortices in these states also have charges $\pm 1 / 4$ as the $s$-wave paired state. Further confirmations are obtained when one computes the shift. If one puts a $d$-wave bosonic condensate on a sphere, without any magnetic field we would have four vortices or antivortices. Since each vortex has charge $1 / 4$, this means 
$\kappa= \pm 1$, which corresponds to $\mathcal{S}=3$ and $\mathcal{S}=-1$ [see Eq. (22)]. These are the values of the shift for the Pfaffian and anti-Pfaffian states, respectively [49].

In real systems, for example, in the $\nu=\frac{5}{2} \mathrm{QH}$ state, whether the $\ell=0$ or $\ell= \pm 2$ is favored is the question about the energy of the ground state and cannot be determined from general principles. It is possible that there is no pairing instability at $\ell=0$, while there are instabilities at finite $\ell$ (Ref. [50]).

\section{E. Electromagnetic response}

In the random phase approximation (RPA), one can relate the response functions of electrons $\Pi_{\mu \nu}$ to the response functions of the Dirac composite fermion, $\tilde{\Pi}_{\mu \nu}$. We use the notation of Eq. (33) and assume the Fermi velocity of the quasiparticles to be much smaller than the speed of light, so the interaction is an instantaneous interaction. The RPA calculation can be done in a straightforward way, and one finds the response functions of the electrons to be

$$
\begin{gathered}
\Pi_{\mathrm{L}}=-\frac{\omega^{2}}{16 \pi^{2} \Delta} \tilde{\Pi}_{\mathrm{L}} \\
\Pi_{\mathrm{T}}=-\frac{\omega^{2}}{16 \pi^{2} \Delta}\left(\tilde{\Pi}_{\mathrm{T}}-\frac{q^{2} V(q)}{16 \pi^{2}}\right), \\
\Pi_{\mathrm{H}}=\frac{\omega^{2}}{16 \pi^{2} \Delta} \tilde{\Pi}_{\mathrm{H}}
\end{gathered}
$$

where

$$
\Delta=\left(\tilde{\Pi}_{\mathrm{T}}-\frac{q^{2} V(q)}{16 \pi^{2}}\right) \tilde{\Pi}_{\mathrm{L}}-\left|\Pi_{\mathrm{H}}\right|^{2}
$$

At $\nu=0$, for massless Dirac composite fermions, we have $\tilde{\Pi}_{H}=0$, and we find a vanishing Hall conductivity of the Dirac electrons, $\Pi_{\mathrm{H}}=0$, as required by $\mathcal{C P}$. The formulas for the other response functions simplify to

$$
\begin{aligned}
& \Pi_{\mathrm{L}}=-\frac{\omega^{2}}{16 \pi^{2} \tilde{\Pi}_{\mathrm{T}}-q^{2} V(q)}, \\
& \Pi_{\mathrm{T}}=-\frac{\omega^{2}}{16 \pi^{2} \tilde{\Pi}_{\mathrm{L}}} .
\end{aligned}
$$

The first equation coincides with the formula in the theory proposed in Ref. [16], but the second does not. The densitydensity correlation function $\Pi_{\mathrm{L}}$ shows the same behavior in the infrared as in the HLR theory. For example, because of the Landau damping in $\tilde{\Pi}_{\mathrm{T}}$ (note that the CFs always have a Fermi surface), $\Pi_{\mathrm{L}}$ has a pole at $\omega \sim-i q^{2}$ for the Coulomb interaction and behaves as $\Pi_{\mathrm{L}} \sim \omega q$ for $\omega \gg q^{2}$, reproducing two key features of the HLR theory [5].
In the limit $q \rightarrow 0$, the formulas simplify considerably. Substituting

$$
\begin{aligned}
& \Pi_{\mathrm{T}}=\Pi_{\mathrm{L}}=\frac{i \omega}{2 \pi} \sigma_{x x}, \\
& \Pi_{\mathrm{H}}=\frac{i \omega}{2 \pi} \sigma_{x y},
\end{aligned}
$$

where $\sigma_{x x}$ and $\sigma_{x y}$ are the longitudinal and Hall conductivities for the electrons, in units of $e^{2} / h=1 / 2 \pi$ (and the version with a tilde for the composite fermions), we find [assuming $V(q)$ diverges slower than $q^{-2}$ at $q \rightarrow 0$ ]

$$
\begin{aligned}
\sigma_{x x} & =\frac{1}{4} \frac{\tilde{\sigma}_{x x}}{\tilde{\sigma}_{x x}^{2}+\tilde{\sigma}_{x y}^{2}}, \\
\sigma_{x y} & =-\frac{1}{4} \frac{\tilde{\sigma}_{x y}}{\tilde{\sigma}_{x x}^{2}+\tilde{\sigma}_{x y}^{2}}+\left[\frac{1}{2}\right]_{\mathrm{NR}} .
\end{aligned}
$$

The term $1 / 2$ on the right-hand side of Eq. (57) should be included in the nonrelativistic case. We concentrate on this case from now on. From Eqs. (56) and (57), we can express $\tilde{\sigma}_{x y}$ in terms of the measurable $\rho_{x x}$ and $\rho_{x y}$ as follows:

$$
\tilde{\sigma}_{x y}=\frac{1}{2}+\frac{\rho_{x y}-2}{\rho_{x x}^{2}+\left(\rho_{x y}-2\right)^{2}} .
$$

Typically, $\rho_{x x}$ is small, and in the regime $\rho_{x y}-2 \sim \rho_{x x}^{2}$, the above equation can be written as

$$
\frac{\rho_{x y}-2}{\rho_{x x}^{2}}=\tilde{\sigma}_{x y}-\frac{1}{2} .
$$

Equation (59) opens up a possibility to experimentally distinguish the Dirac CFs and the standard HLR theory. The HLR theory, as we will argue in Sec. IV, corresponds to $\tilde{\sigma}_{x y}=1 / 2$; thus, the right-hand side of Eq. (59) is zero at exact half filling (e.g., $\rho_{x y}=2$ at exact half filling). On the other hand, for a system near particle-hole symmetry, $\tilde{\sigma}_{x y}$ is small and the right-hand side should be close to $-1 / 2$. One can thus plot the left-hand side of Eq. (59) as a function of the magnetic field $B$ in a range of temperature where $\rho_{x x}$ depends nontrivially on the temperature. Both HLR and our theory then predict that all curves go through one point, whose position on the horizontal axis is the value of the magnetic field at exact half filling $B_{1 / 2}$, and the position on the vertical axis is 0 in the HLR theory and $-1 / 2$ in the theory of the massless Dirac composite fermion. When particle-hole symmetry is not exact, the position of the point on the vertical axis is $-\gamma / 2 \pi$, where $\gamma$ is the Berry phase of the composite fermions around the Fermi disk (assuming Hall conductivity is dominated by the Berry phase). Furthermore, we expect the deviation of $\gamma$ from $\pi$ to be proportional to the amount of Landau-level mixing, i.e., 
the ratio of the Coulomb energy scale and the cyclotron energy. Note that, because of the smallness of $\rho_{x x}$, distinguishing Berry phases of 0 and $\pi$ requires a sufficiently accurate measurement of $\rho_{x y}$ : For a relatively large $\rho_{x x} \sim 0.1 h / e^{2}$, an accuracy better than $5 \times 10^{-3} h / e^{2}$ is needed.

The formulas (56) and (57) are valid at zero frequencies. At finite frequencies, one needs to modify the theory for it to be consistent with Galilean symmetry and Kohn's theorem. The modification and the results for the conductivities are discussed in the Appendix.

\section{F. Infrared divergences}

One can directly check that despite the fact that the dynamical gauge field $a_{\mu}$ does not have a Chern-Simons interaction, the propagator of the transverse component of $a_{i}$ has the same infrared behavior as in the HLR theory:

$$
\left\langle a_{\perp}(0,-\mathbf{q}) a_{\perp}(0, \mathbf{q})\right\rangle \sim \frac{1}{|\mathbf{q}|}
$$

Therefore, we expect that our theory would have the same logarithmic divergences as in the HLR theory with Coulomb interaction.

\section{CONNECTION WITH THE FERMIONIC CHERN-SIMONS THEORY}

The $\mathrm{QH}$ physics of the Dirac fermion shares the same LLL limit with nonrelativistic electrons. The standard theory describing the $\mathrm{FQH}$ states for nonrelativistic electrons is the fermionic CS (HLR) theory. One can then ask the following question: Can one connect the fermionic CS theory and the relativistic theory (36)? This may seem impossible since the former has a CS term in the Lagrangian, while the latter does not. However, we will argue that it is at least possible to go continuously from the relativistic theory to the conventional nonrelativistic fermionic CS theory, if one allows breaking of particle-hole symmetry.

The starting theory of the fermionic CS theory is the Lagrangian

$$
\mathcal{L}=i \psi^{\dagger}\left(\partial_{t}-i A_{0}\right) \psi-\frac{1}{2 m}\left|\left(\partial_{i}-i A_{i}\right) \psi\right|^{2}-\frac{1}{8 \pi} A d A+\cdots
$$

We have included a Chern-Simons term $-(1 / 8 \pi) A d A \equiv$ $-(1 / 8 \pi) \epsilon^{\mu \nu \lambda} A_{\mu} \partial_{\nu} A_{\lambda}$ to take into account the contribution of negative energy states to the Hall conductivity. We now use the standard flux attachment procedure and attach two flux quanta to the fermion $\psi$. The resulting action is then

$$
\begin{aligned}
\mathcal{L}= & i \psi^{\dagger}\left(\partial_{t}-i A_{0}+i c_{0}\right) \psi-\frac{1}{2 m}\left|\left(\partial_{i}-i A_{i}+i c_{i}\right) \psi\right|^{2} \\
& -\frac{1}{8 \pi} A d A+\frac{1}{8 \pi} c d c+\cdots,
\end{aligned}
$$

where $c_{\mu}$ is the statistical gauge field. Now, changing notation,

$$
c_{\mu}=A_{\mu}+2 a_{\mu},
$$

the Lagrangian becomes

$$
\begin{aligned}
\mathcal{L}= & i \psi^{\dagger}\left(\partial_{t}+2 i a_{0}\right) \psi-\frac{1}{2 m}\left|\left(\partial_{i}+2 i a_{i}\right) \psi\right|^{2} \\
& +\frac{1}{2 \pi} a d a+\frac{1}{2 \pi} A d a+\cdots .
\end{aligned}
$$

The form of the action is very similar to Eq. (36), but there are two crucial differences: (i) The $\mathrm{CF}$ is nonrelativistic, and (ii) there is a Chern-Simons interaction $a d a$.

We now suggest that theory (64) can be obtained from Eq. (36) by adding to the latter a $\mathcal{C P}$-breaking mass term and taking the large mass limit. The mass term for the Dirac $\mathrm{CF},-m \bar{\psi} \psi$, is generally expected once one relaxes the condition of particle-hole symmetry (the coefficient of the Chern-Simons term ada cannot change continuously and thus has to remain zero). Now consider the regime where the mass $m$ is very large, and the CF is in the nonrelativistic regime. Let us remind ourselves that if one has a massive Dirac field, coupled to a U(1) gauge field

$$
\mathcal{L}=i \bar{\psi}\left(\partial_{\mu}-i a_{\mu}\right) \psi-m \bar{\psi} \psi+\mu \bar{\psi} \gamma \gamma^{0} \psi,
$$

and if the chemical potential is inside the gap, i.e., $|\mu|<|m|$, then one can integrate out $\psi$ to obtain an effective action for $a_{\mu}[51,52]$,

$$
-\frac{1}{8 \pi} \frac{m}{|m|} \text { ada. }
$$

Now imagine that $\mu$ is slightly above the mass, $\mu>|m|$ but $\mu-|m| \ll m$. In this case, one has to take into account the gapless fermions, which are nonrelativistic, but one should also not forget the induced Chern-Simons term (66), which can be thought of as coming from the filled Dirac sea and is largely unaffected by a small density of added fermions. Hence, low-energy dynamics is described by

$$
\begin{aligned}
\mathcal{L}= & i \psi^{\dagger}\left(\partial_{t}-i a_{0}\right) \psi-\frac{1}{2|m|}\left|\left(\partial_{i}-i a_{i}\right)^{2} \psi\right|^{2} \\
& -\frac{1}{8 \pi} \frac{m}{|m|} a d a+\cdots
\end{aligned}
$$

By the substitution $a \rightarrow-2 a$, and assuming $m<0$, one obtains the action (64) before coupling to 
electromagnetism. Thus, the usual fermionic CS theory is obtained in a large mass, nonrelativistic limit of the Dirac CF theory. In other words, one can deform the Dirac CF theory without a CS term into the conventional nonrelativistic theory with a CS term, at the price of breaking particle-hole symmetry.

This does not mean that we have been able to derive the effective field theory (36) using the familiar fluxattachment procedure, but now we can look at the old puzzle of Ref. [15] in a new light. If one performs the RPA calculation using the action (64), we would find expressions identical to Eqs. (48)-(50) with the replacement

$$
\tilde{\Pi}_{\mathrm{H}} \rightarrow \frac{i \omega}{2 \pi}\left(\sigma_{x y}^{\mathrm{CF}}+\frac{1}{2}\right),
$$

where $\sigma_{x y}^{\mathrm{CF}}$ is the Hall conductivity of the CFs (as a function of frequency and wave number) and $\frac{1}{2}$ comes from the Chern-Simons term ada. To be consistent with Eq. (34), $\sigma_{x y}^{\mathrm{CF}}$ must be equal to $-\frac{1}{2}$ for all frequencies and wave numbers. However, at least in the regime $\omega \gg v_{F} q$, the Hall conductivity of a Fermi liquid is expected to be zero. This is essentially the puzzle identified in Ref. [15], and this puzzle would not exist if the low-energy dynamics is indeed described by the Dirac composite fermion. For the latter, the nonzero value of $\sigma_{x}^{\mathrm{CF}}$ can be interpreted as coming from the Berry phase of the CF around the Fermi disk. It seems that the key to understanding the particle-hole symmetry in the composite fermion theory is to understand the appearance of this Berry phase.

\section{CONCLUSIONS}

We have presented evidence that the composite fermion of the half-filled Landau level is a Dirac fermion, whose Dirac mass vanishes when particle-hole symmetry is exact. Although we did not try to derive Eq. (36) microscopically, it is hoped that such a derivation is possible, following one of the methods used in Ref. [39] for deriving the composite Dirac-liquid Hamiltonian. We also notice that the LLL Lagrangian (9) suggests that the Dirac spinor may involve, as one of its components, the Lagrange multiplier $\chi$ that enforces the LLL constraints. For now, it is also clear that the conventional flux attachment procedure cannot be applied to the quantum Hall effect for a Dirac fermion in a particle-hole symmetric manner.

The proposed map between the electromagnetic current in the original and low-energy effective theory, $\bar{\Psi} \gamma^{\mu} \Psi=(1 / 2 \pi) \epsilon^{\mu \nu \lambda} \partial_{\nu} a_{\lambda}$, is reminiscent of mirror symmetry [53] in three dimensions. Recently, Hook et al. used mirror symmetry to argue that the ground state of $(2+1)$ dimensional supersymmetric QED in the presence of magnetic impurities is characterized by an emergent Fermi surface which encloses a volume proportional to the magnetic field [54]. The emergent fermion is a fermionic vortex which was interpreted as a bound state of a gaugino with a dual photon. It is tempting to speculate a connection between mirror symmetry and the physics of the half-filled Landau level.

We have suggested that careful measurement of transport near half filling can determine the Berry phase of the composite fermion around the Fermi surface, and it distinguishes the standard HLR scenario and the scenario of Dirac CF. Our theory implies the existence of a new gapped phase, the PH-Pfaffian state, distinct from the Pfaffian and anti-Pfaffian states by being its own particle-hole conjugate. It would be interesting to see if there exist physical systems where such a phase is realized. Besides GaAs and the surface of 3D TIs, systems with a tunable parameter like bilayer graphene in a perpendicular magnetic field $[55,56]$ seem to be promising venues to search for this new phase.

\section{ACKNOWLEDGMENTS}

The author thanks Alexander Abanov, Clay Córdova, Eduardo Fradkin, Siavash Golkar, Jainendra Jain, Woowon Kang, Michael Levin, Eun-Gook Moon, Sergej Moroz, Sungjay Lee, Andrei Parnachev, Xiao-Liang Qi, Subir Sachdev, Steve Simon, Boris Spivak, and Paul Wiegmann for valuable discussions and comments. This work is supported, in part, by the U.S. DOE Grant No. DEFG02-13ER41958, MRSEC Grant No. DMR-1420709 by the NSF, ARO MURI Grant No. 63834-PH-MUR, and by a Simons Investigator grant from the Simons Foundation. The author thanks Maissam Barkeshli and Michael Mulligan for illuminating discussions.

Note added.-After this work was completed, the author became aware of Ref. [57], which has some overlap with the current paper. In particular, the phase $\mathrm{CFL}_{2}$ in Fig. 5 of Ref. [57] differs from the picture advocated here only by the presence of a second Fermi surface, coupled to $a_{\mu}$ and carrying a trivial Berry phase. In addition, Ashwin Vishwanath informed the author that the topological order of the PH-Pfaffian phase has been previously proposed within the context of topological superconductors [58].

\section{APPENDIX: GALILEAN INVARIANCE AND KOHN'S THEOREM}

In the main text, we have avoided the issue of Galilean invariance and the related Kohn's theorem. Kohn's theorem states that in the absence of disorder, the $q=0$, finitefrequency electromagnetic response of a system of interacting nonrelativistic electrons is the same as of noninteracting electrons. In particular, the only pole in the response is at the cyclotron frequency. In the LLL limit $m \rightarrow 0$, the cyclotron frequency is infinite, and the response is especially simple: $\sigma_{x x}(\omega)=0, \sigma_{x y}(\omega)=\nu$ (the conductivities are measured in units of $\left.e^{2} / h=1 / 2 \pi\right)$. On the other hand, Eq. (56) gives nonzero $\sigma_{x x}$ at finite $\omega$. The problem 
stems from the fact that the theory we were using does not have the Galilean invariance of the original electrons.

The full solution to the problem of Galilean invariance, capable of reproducing the more subtle effects such as the $q^{2}$ dependence of the Hall conductivity of gapped states in the clean limit $[59,60]$, will be presented elsewhere. Here, we only sketch a simplified version of the theory, adequate for finding the $q=0$, finite-frequency response. What follows is, in a sense, a version (streamlined and trivially adapted to Dirac CFs) of the phenomenological "modified RPA" (MRPA) proposal of Ref. [61]. (At $q=0$, one does not need to be concerned with the issues that have led to the "MMRPA" scheme of Ref. [62].)

Galilean invariance is implemented by the introduction of a dynamic field $v^{i}$, which transforms as a velocity under Galilean boosts. The effective Lagrangian is schematically

$$
\mathcal{L}=\mathcal{L}\left(\psi, \psi^{\dagger}, a_{\mu}, v^{i}\right)+\frac{1}{2 \pi} \epsilon^{\mu \nu \lambda} A_{\mu} \partial_{\nu} a_{\lambda} .
$$

For modes near the Fermi surface, the coupling of $v^{i}$ to other fields can be subsumed into a modified gauge potential,

$$
\begin{aligned}
& \tilde{a}_{0}=a_{0}-\frac{1}{4} m_{*} v^{2}, \\
& \tilde{a}_{i}=a_{i}+\frac{1}{2} m_{*} v_{i},
\end{aligned}
$$

where $m_{*}$ is the effective mass of the composite fermions on the Fermi surface. In terms of $\tilde{a}_{\mu}$, the Lagrangian is approximately

$$
\mathcal{L}=\mathcal{L}\left(\psi, \psi^{\dagger}, \tilde{a}_{\mu}\right)+\frac{1}{2 \pi} \epsilon^{\mu \nu \lambda} A_{\mu} \partial_{\nu} a_{\lambda},
$$

where $\psi$ has a gauge coupling with $\tilde{a}_{\mu}$ with charge -2 . (Note that the mixed Chern-Simons term still involves $a_{\mu}$, not $\tilde{a}_{\mu}$.) One can integrate out $v^{i}$ to obtain interactions between the composite fermions, the major part of which is a Landautype interaction required to restore Galilean invariance. To perform calculations in the RPA, it is, however, more convenient to leave $v^{i}$ in the Lagrangian.

Differentiating the action with respect to $a_{\mu}$, we get the relationship between the composite fermion density and the current with the external field,

$$
\rho_{\mathrm{CF}}=\frac{B}{4 \pi}, \quad \mathbf{j}_{\mathrm{CF}}=\frac{\mathbf{E} \times \hat{\mathbf{z}}}{4 \pi} .
$$

Differentiating with respect to $v^{i}$, one gets, on the other hand, $\mathbf{j}_{\mathrm{CF}}=\rho_{\mathrm{CF}} \mathbf{v}$, which implies

$$
\mathbf{v}=\frac{\mathbf{E} \times \hat{\mathbf{z}}}{B} .
$$

Denoting by $\tilde{\sigma}^{i j}$ the conductivity tensor of the CFs, we have, for the composite fermion current,

$$
j_{\mathrm{CF}}^{i}=\frac{\tilde{\sigma}^{i j}}{2 \pi}\left(-2 \tilde{e}_{j}\right)=\frac{\tilde{\sigma}^{i j}}{2 \pi}\left(-2 e_{j}+m_{*} \dot{v}_{j}\right) .
$$

Furthermore, the physical electromagnetic current is related to the field tensor constructed from $a_{\mu}$ [Eq. (41)]. From the formulas listed above, one can get the conductivities of the electrons,

$$
\begin{aligned}
& \sigma_{x x}=\frac{1}{4} \frac{\tilde{\sigma}_{x x}}{\tilde{\sigma}_{x x}^{2}+\tilde{\sigma}_{x y}^{2}}+\frac{m_{*}}{2 B} i \omega, \\
& \sigma_{x y}=-\frac{1}{4} \frac{\tilde{\sigma}_{x y}}{\tilde{\sigma}_{x x}^{2}+\tilde{\sigma}_{x y}^{2}}+\left[\frac{1}{2}\right]_{\mathrm{NR}} .
\end{aligned}
$$

One sees that the only modification compared to Eqs. (56) and (57) is the appearance of an additional term linear in $\omega$ on the right-hand side of Eq. (A8a).

It is instructive to consider the Drude model for the composite fermions, parametrizing disorders by a single relaxation time $\tau$. In this approximation, the conductivities of the CFs are

$$
\begin{aligned}
& \tilde{\sigma}_{x x}=2 \pi \rho_{\mathrm{CF}} \frac{m_{*}\left(-i \omega+\tau^{-1}\right)}{m_{*}^{2}\left(-i \omega+\tau^{-1}\right)^{2}+4 b^{2}}, \\
& \tilde{\sigma}_{x y}=-2 \pi \rho_{\mathrm{CF}} \frac{2 b}{m_{*}^{2}\left(-i \omega+\tau^{-1}\right)^{2}+4 b^{2}} .
\end{aligned}
$$

Substituting these expressions into Eqs. (A8), we find remarkably simple expressions,

$$
\begin{aligned}
\sigma_{x x} & =\frac{m_{*}}{2 B \tau}, \\
\sigma_{x y} & =\frac{b}{B}+\frac{1}{2}=\nu .
\end{aligned}
$$

In the relaxation time approximation, the electron conductivities do not depend on frequency, and the Hall conductivity is at its classical value. The clean case can be recovered by taking $\tau \rightarrow \infty$. In this case, $\sigma_{x x}=0$ and $\sigma_{x y}=\nu$, as required by Kohn's theorem at $m=0$.

[1] D. C. Tsui, H. L. Stormer, and A. C. Gossard, TwoDimensional Magnetotransport in the Extreme Quantum Limit, Phys. Rev. Lett. 48, 1559 (1982).

[2] R. B. Laughlin, Anomalous Quantum Hall Effect: An Incompressible Quantum Fluid with Fractionally Charged Excitations, Phys. Rev. Lett. 50, 1395 (1983).

[3] J. K. Jain, Composite-Fermion Approach for the Fractional Quantum Hall Effect, Phys. Rev. Lett. 63, 199 (1989).

[4] A. Lopez and E. Fradkin, Fractional Quantum Hall Effect and Chern-Simons Gauge Theories, Phys. Rev. B 44, 5246 (1991). 
[5] B. I. Halperin, P. A. Lee, and N. Read, Theory of the HalfFilled Landau Level, Phys. Rev. B 47, 7312 (1993).

[6] J. K. Jain, Composite Fermions (Cambridge University Press, Cambridge, England, 1997).

[7] R. L. Willett, M. A. Paalanen, R. R. Ruel, K. W. West, L. N. Pfeiffer, and D. J. Bishop, Anomalous Sound Propagation at $\nu=\frac{1}{2}$ in a $2 D$ Electron Gas: Observation of a Spontaneously Broken Translational Symmetry?, Phys. Rev. Lett. 65, 112 (1990).

[8] W. Kang, H. L. Stormer, L. N. Pfeiffer, K. W. Baldwin, and K. W. West, How Real Are Composite Fermions?, Phys. Rev. Lett. 71, 3850 (1993).

[9] V. J. Goldman, B. Su, and J. K. Jain, Detection of Composite Fermions by Magnetic Focusing, Phys. Rev. Lett. 72, 2065 (1994).

[10] G. W. Moore and N. Read, Nonabelions in the Fractional Quantum Hall Effect, Nucl. Phys. B360, 362 (1991).

[11] N. Read and D. Green, Paired States of Fermions in Two Dimensions with Breaking of Parity and Time-Reversal Symmetries and the Fractional Quantum Hall Effect, Phys. Rev. B 61, 10267 (2000).

[12] S. M. Girvin, Particle-Hole Symmetry in the Anomalous Quantum Hall Effect, Phys. Rev. B 29, 6012 (1984).

[13] M. Levin, B. I. Halperin, and B. Rosenow, Particle-Hole Symmetry and the Pfaffian State, Phys. Rev. Lett. 99, 236806 (2007).

[14] S. S. Lee, S. Ryu, C. Nayak, and M. P. A. Fisher, ParticleHole Symmetry and the $\nu=\frac{5}{2}$ Quantum Hall State, Phys. Rev. Lett. 99, 236807 (2007).

[15] S. A. Kivelson, D.-H. Lee, Y. Krotov, and J. Gan, Composite-Fermion Hall Conductance at $\nu=\frac{1}{2}$, Phys. Rev. B 55, 15552 (1997).

[16] D.-H. Lee, Neutral Fermions at Filling Factor $\nu=1 / 2$, Phys. Rev. Lett. 80, 4745 (1998).

[17] E. H. Rezayi and F. D. M. Haldane, Incompressible Paired Hall State, Stripe Order, and the Composite Fermion Liquid Phase in Half-Filled Landau Levels, Phys. Rev. Lett. 84, 4685 (2000).

[18] X. G. Wu, G. Dev, and J. K. Jain, Mixed-Spin Incompressible States in the Fractional Quantum Hall Effect, Phys. Rev. Lett. 71, 153 (1993).

[19] D. Kamburov, Y. Liu, M. A. Mueed, M. Shayegan, L. N. Pfeiffer, K. W. West, and K. W. Baldwin, What Determines the Fermi Wave Vector of Composite Fermions?, Phys. Rev. Lett. 113, 196801 (2014).

[20] K. S. Novoselov, A. K. Geim, S. V. Morozov, D. Jiang, M. I. Katsnelson, I. V. Grigorieva, S. V. Dubonos, and A. A. Firsov, Two-Dimensional Gas of Massless Dirac Fermions in Graphene, Nature 438, 197 (2005).

[21] Y. Zhang, Y.-W. Tan, H. L. Stormer, and P. Kim, Experimental Observation of the Quantum Hall Effect and Berry's Phase in Graphene, Nature 438, 201 (2005).

[22] X. Du, I. Skachko, F. Duerr, A. Luican, and E. Y. Andrei, Fractional Quantum Hall Effect and Insulating Phase of Dirac Electrons in Graphene, Nature 462, 192 (2009).

[23] K. I. Bolotin, F. Ghahari, M. D. Shulman, H. L. Stormer, and P. Kim, Observation of the Fractional Quantum Hall Effect in Graphene, Nature 462, 196 (2009).

[24] J. G. Analytis, R. D. McDonald, S. C. Riggs, J.-H. Chu, G. S. Boebinger, and I. R. Fisher, Two-Dimensional Surface
State in the Quantum Limit of a Topological Insulator, Nat. Phys. 6, 960 (2010).

[25] D. V. Khveshchenko, Composite Dirac Fermions in Graphene, Phys. Rev. B 75, 153405 (2007).

[26] Note that in Read's LLL theory of the bosonic $\nu=1$ state [27] there is also a gauge field with no Chern-Simons term.

[27] N. Read, Lowest-Landau-Level Theory of the Quantum Hall Effect: The Fermi-Liquid-Like State of Bosons at Filling Factor One, Phys. Rev. B 58, 16262 (1998).

[28] F. D. M. Haldane, Berry Curvature on the Fermi Surface: Anomalous Hall Effect as a Topological Fermi-Liquid Property, Phys. Rev. Lett. 93, 206602 (2004).

[29] D. X. Nguyen, D. T. Son, and C. Wu, Lowest Landau Level Stress Tensor and Structure Factor of Trial Quantum Hall Wave Functions, arXiv:1411.3316.

[30] J. Martínez and M. Stone, Current Operators in the Lowest Landau Level, Int. J. Mod. Phys. B 07, 4389 (1993).

[31] R. Rajaraman and S. L. Sondhi, Landau Level Mixing and Solenoidal Terms in Lowest Landau Level Currents, Mod. Phys. Lett. B 08, 1065 (1994).

[32] S. Golkar, M. M. Roberts, and D. T. Son, Effective Field Theory of Relativistic Quantum Hall Systems, J. High Energy Phys. 12 (2014) 138.

[33] S. Deser, R. Jackiw, and S. Templeton, Topologically Massive Gauge Theories, Ann. Phys. (N.Y.) 140, 372 (1982).

[34] The parity anomaly [51,52] is not relevant for the current problem, as the gauge field propagates in $(3+1)$ dimensions [35].

[35] M. Mulligan and F. J. Burnell, Topological Insulators Avoid the Parity Anomaly, Phys. Rev. B 88, 085104 (2013).

[36] M. Levin and D. T. Son (unpublished).

[37] N. Read, Theory of the Half-Filled Landau Level, Semicond. Sci. Technol. 9, 1859 (1994).

[38] N. Read, Recent Progress in the Theory of Composite Fermions near Even-Denominator Filling Factors, Surf. Sci. 361-362, 7 (1996).

[39] D. F. Mross, A. Essin, and J. Alicea, Composite Dirac Liquids: Parent States for Symmetric Surface Topological Order, Phys. Rev. X 5, 011011 (2015).

[40] C. Wang, A. C. Potter, and T. Senthil, Gapped Symmetry Preserving Surface State for the Electron Topological Insulator, Phys. Rev. B 88, 115137 (2013).

[41] P. Bonderson, C. Nayak, and X.-L. Qi, A Time-Reversal Invariant Topological Phase at the Surface of a 3D Topological Insulator, J. Stat. Mech. (2013) P09016.

[42] X. Chen, L. Fidkowski, and A. Vishwanath, Symmetry Enforced Non-Abelian Topological Order at the Surface of a Topological Insulator, Phys. Rev. B 89, 165132 (2014).

[43] M. A. Metlitski, C. L. Kane, and M. P. A. Fisher, A Symmetry-Respecting Topologically-Ordered Surface Phase of 3D Electron Topological Insulators, arXiv:1306.3286.

[44] G. Baym and S. A. Chin, Landau Theory of Relativistic Fermi Liquids, Nucl. Phys. A262, 527 (1976).

[45] J. Polchinski, Effective Field Theory, and the Fermi Surface, arXiv:hep-th/9210046.

[46] R. Shankar, Renormalization Group Approach to Interacting Fermions, Rev. Mod. Phys. 66, 129 (1994).

[47] M. P. A. Fisher and D. H. Lee, Correspondence between Two-Dimensional Bosons and a Bulk Superconductor in a Magnetic Field, Phys. Rev. B 39, 2756 (1989). 
[48] X. G. Wen and A. Zee, Shift and Spin Vector: New Topological Quantum Numbers for the Hall Fluids, Phys. Rev. Lett. 69, 953 (1992).

[49] If one would want to make the anti-Pfaffian state in the conventional CF theory, to match the shift, one would have to pair the CFs in an $f$-wave channel $\ell=-3$, compared to $\ell=+1$ for the Pfaffian state.

[50] W. Kohn and J. M. Luttinger, New Mechanism for Superconductivity, Phys. Rev. Lett. 15, 524 (1965).

[51] A. J. Niemi and G. W. Semenoff, Axial-Anomaly-Induced Fermion Fractionization and Effective Gauge-Theory Actions in Odd-Dimensional Space-Times, Phys. Rev. Lett. 51, 2077 (1983).

[52] A. N. Redlich, Parity Violation, and Gauge Noninvariance of the Effective Gauge Field Action in Three Dimensions, Phys. Rev. D 29, 2366 (1984).

[53] K. A. Intriligator and N. Seiberg, Mirror Symmetry in ThreeDimensional Gauge Theories, Phys. Lett. B 387, 513 (1996).

[54] A. Hook, S. Kachru, G. Torroba, and H. Wang, Emergent Fermi Surfaces, Fractionalization, and Duality in Supersymmetric QED, J. High Energy Phys. 08 (2014) 031.

[55] K. Lee, B. Fallahazad, J. Xue, D. C. Dillen, K. Kim, T. Taniguchi, K. Watanabe, and E. Tutuc, Chemical Potential and Quantum Hall Ferromagnetism in Bilayer Graphene, Science 345, 58 (2014).

[56] P. Maher et al., Tunable Fractional Quantum Hall Phases in Bilayer Graphene, Science 345, 61 (2014).

[57] M. Barkeshli, M. Mulligan, and M. P. A. Fisher, ParticleHole Symmetry and the Composite Fermi Liquid, arXiv:1502.05404.

[58] L. Fidkowski, X. Chen, and A. Vishwanath, Non-Abelian Topological Order on the Surface of a 3D Topological Superconductor from an Exactly Solved Model, Phys. Rev. X 3, 041016 (2013).

[59] C. Hoyos and D. T. Son, Hall Viscosity and Electromagnetic Response, Phys. Rev. Lett. 108, 066805 (2012).

[60] B. Bradlyn, M. Goldstein, and N. Read, Kubo Formulas for Viscosity: Hall Viscosity, Ward Identities, and the Relation with Conductivity, Phys. Rev. B 86, 245309 (2012).

[61] S. H. Simon and B. I. Halperin, Finite-Wave-Vector Electromagnetic Response of Fractional Quantum Hall States, Phys. Rev. B 48, 17368 (1993).

[62] S. H. Simon, A. Stern, and B. I. Halperin, Composite Fermions with Orbital Magnetizations, Phys. Rev. B 54, R11114 (1996). 